\title{
Numerical simulation of fluid-structure interaction between elastic thin-walled structure and transient fluid flow
}

\author{
Evgeny I. Boznyakov ${ }^{1,3^{*}}$, Irina N. Afanasyeva ${ }^{1,2}$, and Alexander M. Belostotsky ${ }^{1,2}$ \\ ${ }^{1}$ Moscow State University of Civil Engineering, Research\&Educational Center of Computational \\ Simulation, 26 Yaroslavskoye Shosse, Moscow, 129337, Russia \\ ${ }^{2}$ Research \& Development Centre StaDyO Ltd, Office 810, 18, 3-ya Ulitsa Yamskogo Polya, \\ Moscow, 125040, Russia \\ ${ }^{3}$ «PIK-Proekt» Ltd., Moscow, Russia
}

\begin{abstract}
The present paper deals with the verification of a numerical simulation technique of fluid-structure interaction (FSI) problems. The configuration of the task consists of viscous incompressible fluid around an elastic cylindrical thin-walled structure where the external flow is fully turbulent (Reynolds numbers $\mathrm{Re}=1.2 \cdot 10^{7}$ ). The verification is divided into three stages: 1) verification of the mechanical finite element model, 2) verification of the fluid finite volume model and 3) verification of the Fluid-Structure interaction model. Within the stage 1 the comparison of the obtained results with the full-scale measurements and the results of the alternative numerical simulations are presented below.
\end{abstract}

\section{Introduction}

Structures of cylindrical thin-walled shells with a certain combination of a specific geometrical parameters, physical, mechanical and dynamic properties of the system and the environment are sensitive to wind effects. Because of the interaction between consider structures and wind flow certain aeroelastic phenomena appeared due to the vortex shedding can be observed (oscillations of a thin-walled cylindrical shell under the flow excitation).

Considering dissipative dynamic system "flow-structure", it should pay attention to the range of natural frequencies and modes of structure and the main frequency of the vortex shedding. In the case of their proximity it leads to significant displacement with increasing amplitude of thin-walled shell oscillations which leads to a change of boundary conditions.

An assessment of the mutual influence of wind gusts on the structure and the deformed structure on the spatial and temporal characteristics of the flow is possible only using a modern numerical models and methods that lets to solve multidisciplinary coupled problems.

* Corresponding author: evgenyboznyakov@gmail.com 


\section{Problem statement}

In the development of the numerical simulation technique for solving three-dimensional aerohydroelasticity problems in civil engineering [4] as well as to examine in detail the nature of aeroelastic vibrations of thin-walled cylindrical structures the problem of numerical simulation of the empty silo oscillations induced by external flow was selected. This verification example is based on the fact that in October 2002 at the port of Antwerp (Belgium) during the storm the oscillations of several empty silos from a group of forty ones was observed $[1,2]$.

The verification task is divided into three stages. The first stage is the verification of finite element models of a thin-walled cylindrical tank based on a modal analysis and comparison of the obtained results with full-scale measurements and the results of alternative numerical simulations. The second one is the verification of the fluid finite volume model based on the numerical solution of the equations of the Navier-Stokes equations for incompressible Newtonian fluid using a turbulence model URANS SST. Comparison of the obtained results is occurred with data from alternative numerical simulations $[1,2]$. The third stage - the verification of the aeroelastic model, involves the determination of the spatial and temporal characteristics of the fluid flow and the stressstrain state of structures in time using FSI technology (Fluid-Structure Interaction). Within this stage the obtained results compared with data from $[1,2]$.

As a primary tool for performing a numerical simulation of the fluid-structure interaction problems the specialized multidisciplinary software ANSYS (Mechanical and CFX) was chosen.

\section{Stage 1: Verification of the mechanical finite element model}

\subsection{Description of the computational models}

The object of study is one of the forty silos located in 5 rows of 8 each. The distance between them is $30 \mathrm{~cm}$ (Fig. 1). Cylindrical shell with a diameter of $5.5 \mathrm{~m}$ and a height of $25 \mathrm{~m}$ consists of 10 aluminum plates with a height of $2.5 \mathrm{~m}$ and a thickness decreasing with a height (Fig. 1, Table 1).
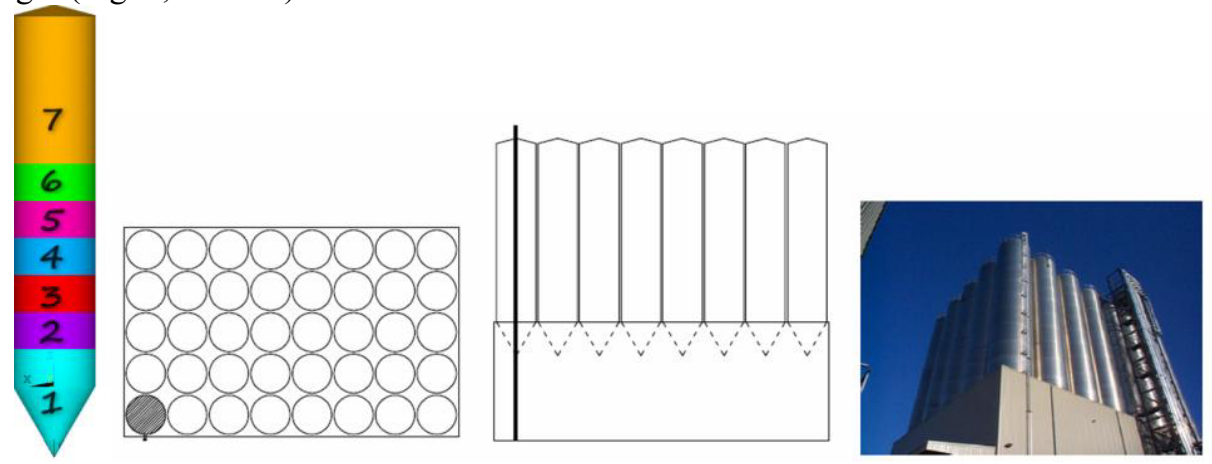

Fig. 1. Geometrical model of the silo (ANSYS Mechanical, different shell thickness marked with color and number), plan, lateral view and picture of the silo group in the port of Antwerp (Belgium). 
Table 1. Variation of the shell thickness of the cylindrical silo along the height

\begin{tabular}{|c|c|c|}
\hline $\begin{array}{c}\text { Height, } \\
{[\mathbf{m}]}\end{array}$ & $\begin{array}{c}\text { Thickness, } \\
{[\mathbf{m m}]}\end{array}$ & $\begin{array}{c}\text { Numbers } \\
\text { matching } \\
\text { (Fig.1) }\end{array}$ \\
\hline $0.0-2.5$ & 10.5 & $(1)$ \\
\hline $2.5-5.0$ & 9.0 & $(2)$ \\
\hline $5.0-7.5$ & 8.5 & $(3)$ \\
\hline $7.5-10.0$ & 7.5 & $(4)$ \\
\hline $10.0-12.5$ & 7.0 & $(5)$ \\
\hline $12.5-15.0$ & 6.5 & $(6)$ \\
\hline $15.0-25.0$ & 6.0 & $(7)$ \\
\hline
\end{tabular}

The parametric three-dimensional shell finite-element (FE) model of the silo was created in the ANSYS Mechanical preprocessor. Type element SHELL181 was used that is dictated by the structure geometry. It is four-node element with six degrees of freedom at each node that implements Mindlin-Reissner shell theory.

The cones at an angle of $15^{\circ}$ and $60^{\circ}$ are welded to the top and bottom of the cylinder respectively. Perpendicular beam-pillars support the lower part of the cylinder and fastened at four points on the circle. For which of them the appropriate boundary conditions in the FE model are set: all linear $(\mathrm{UX}=\mathrm{UY}=\mathrm{UZ}=0)$ and angular $(\mathrm{ROTX}=\mathrm{ROTX}=\mathrm{ROTZ}=0)$ displacements are limited at the four nodes where the silo connects with support beams.

Table 2. FE computational grids characteristics

\begin{tabular}{|c|c|c|c|c|c|}
\hline Model & $\begin{array}{c}\text { Elements } \\
\text { number }\end{array}$ & $\begin{array}{c}\text { Nodes } \\
\text { number }\end{array}$ & $\begin{array}{c}\text { Elements number } \\
\text { along the } \\
\text { ccircumference }\end{array}$ & $\begin{array}{c}\text { Vertical } \\
\text { elements } \\
\text { number }\end{array}$ & $\begin{array}{c}\text { Ratio of the } \\
\text { recommended } \boldsymbol{h}_{\boldsymbol{e}} \text { to } \\
\text { factual one }\end{array}$ \\
\hline D1 & 1686 & 1680 & 60 & 20 & 48.64 \\
\hline M1 & 4142 & 4142 & 60 & 52 & 11.15 \\
\hline M2 & 15000 & 15002 & 120 & 80 & 2.23 \\
\hline M3 & 60000 & 60002 & 240 & 160 & 1.00 \\
\hline
\end{tabular}

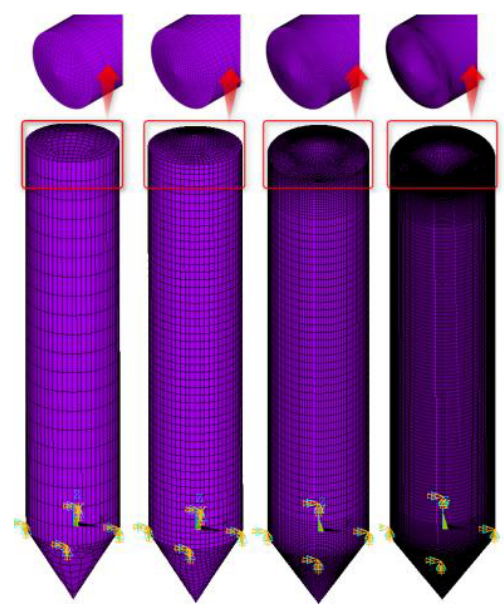

Fig.2. FE models of the single silo (ANSYS Mechanical, SHELL181) with an indication of the boundary conditions (Models D1, M1, M2, M3). 
To study the grid convergence for four types of the computational grid (Fig. 2, tab. 2) with a successive increasing of an elements number were tested. In the areas of the shell edge effect (the joints "floor-wall", "wall-cover") FE vertical size $h_{e}$ was chosen according the condition of an adequate definition of the torque components:

$$
h_{e} \leq 0,2 \sqrt{R \cdot t h}
$$

where $R=2.75[\mathrm{~m}]$ - the radius of the silo; $t h=0.006$ [m] - wall thickness of the silo.

\subsection{Simulation parameters}

The following physical and mechanical properties of FE models were set: Young's modulus $\mathrm{E}=6,7610^{4} \mathrm{MPa}$, density $\rho=2700 \mathrm{~kg} / \mathrm{m}^{3}$ and Poisson's ratio $v=0,35$ corresponding to the material (aluminum) of the structural elements of the silo.

Modal analysis was conducted in ANSYS Mechanical using Block Lanczos method [3]. Sparse direct solver is used for solving the linear algebraic equations.

Mode shapes are characterized by a pair of parameters $(m, n)$, where $m$ is a number of half waves in a longitudinal (along a height) direction of the shell, $n$ is a number of circumferential waves of the shell. Data of the field tests are taken from the electronic source [1]. Vibration measurements were conducted on the corner silo, where the maximum amplitude of vibration under the strong wind excitation was detected. The sensors were located at various points along the height. The radial acceleration was measured at 10 points.

\subsection{Results and conclusions for the stage 1}

The Figure 3 illustrates the numerical results of the stage 1: natural frequencies and mode shapes of a single silo in comparison with numerical and experimental reference data $[1,2]$ and the relative errors calculated according to the formula (2):

$$
\varepsilon=\frac{\left|x_{\text {ref }}-x_{\text {calc }}\right|}{x_{\text {ref }}} \cdot 100 \%,
$$

where $x_{r e f}$ - reference data $[1,2]$ and $x_{\text {calc }}$ - the results of numerical calculations performed by the authors of the present article.

The grid convergence for four types of the computational grid is demonstrated. 

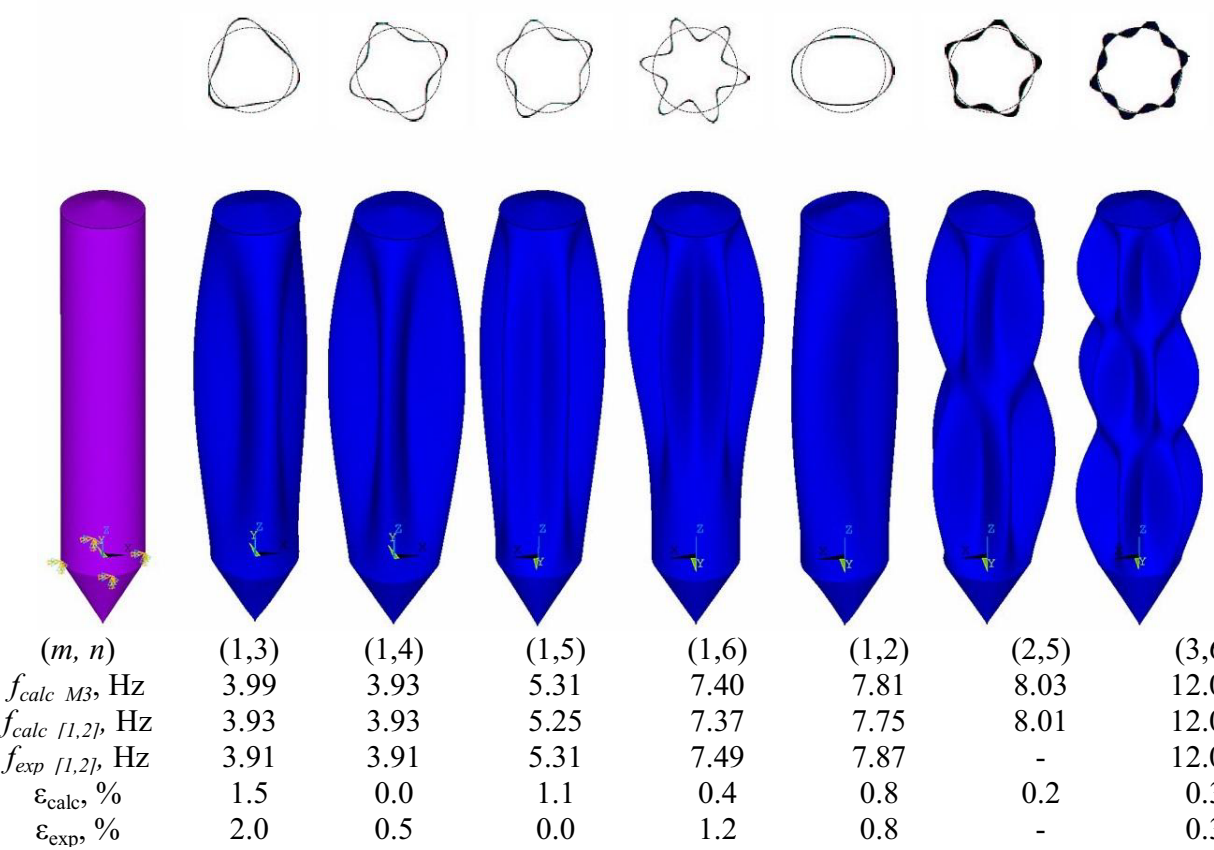

$(1,3)$

$(1,4)$

$(1,5)$

$(1,6)$

5.31

5.25

3.93

7.40

$(1,2)$

$(2,5)$

$(3,6)$

7.37

5.31

7.49

7.81

7.75

3.91

1.1

0.4

0.0
0.5

0.0

7.87

8.03

12.04

$8.01 \quad 12.00$

1.5

2.0

1.2

0.8

12.00

0.2

0.3

0.8

0.3

Fig.3. The mode shapes and corresponding natural frequencies of the single silo with an indication of the relative error $\varepsilon$. First row - reference numerical results [1,2], second row - obtained numerical results (Model M3).

The comparison with experiment shows the expected smallness of error for the best series of experiments. The discrepancy is convincingly explained by errors of the experiment (first of all by the imperfection of the cylindrical shape). At the same time the high proximity of the obtained numerical results to the numerical results presented in reference $[1,2]$ is observed.

Taking into account the numerical results of aerodynamic studies from [1, 2] and data from domestic codes [5], we can estimate the main vortex shedding frequency $\left(f_{v s}\right)$ for cylindrical structures is in the range from $1.56 \mathrm{~Hz}$ to $2.13 \mathrm{~Hz}$. As shown by numerical simulations and experiments the closest natural frequencies of considering cylindrical structure to these range of frequencies are those that correspond to $m=1, n=3$ and $n=4$ $(f(1,3)=4.06 \mathrm{~Hz}, f(1,4)=4.00 \mathrm{~Hz})$. The effect of other modes (the nearest one $f(1,5)=$ $5.50 \mathrm{~Hz}$ ) can be neglected.

For further FSI investigations the "coarse" FE model D1 is chosen because it provides an acceptable accuracy and computational efficiency so necessary for solving nonlinear dynamic coupled problems.

\section{Summary}

Within the stage 1 the comparison of the obtained results with the full-scale measurements and the results of the alternative numerical simulations [1,2] were carried out. For further FSI investigations the main FE-model are chosen.

As a next step of study it is planning to perform the verification of the fluid finite volume model (stage 2). Outcomes of $2 \mathrm{D}$ - and 3D-simulations will present. The grid convergence for several types of the computational grid will demonstrate. The comparison with the alternative numerical simulations $[1,2]$ will conduct. For further FSI investigations the main FV-model will chosen. 
The third stage will be devoted to the numerical simulation of the thin-walled silo behavior under transient wind acting. The values of radial displacements at selected points located halfway up the height of the cylindrical part of the silo will compare with the alternative numerical simulations [1,2]. The assessment of the mutual influence of wind gusts on the structure and the deformed structure on the spatial and temporal characteristics of the fluid flow will study.

The Reported study was funded by Ministry of Education and Science of the Russian Federation (RF President Grant, agreement №14.Z56.16.8493-MK).

\section{References}

1. D. Dooms, G. Degrande, De G. Roeck and E. Reynders, Wind induced vibration of thin-walled cylindrical structures, Proceedings for ISMA2004, 781-796 (2004).

2. D. Dooms, Fluid structure interaction applied to flexible silo constructions, $\mathrm{PhD}$ thesis (2009).

3. Mark T. Jones, Merrell L. Patrick, The use of Lanczos's method to solve the large generalized symmetric definite eigenvalue problem, NASA Contractor report 181914 ICASE Report 89-69 (1989).

4. I.N. Afanasyeva, Adaptivnaya metodika chislennogo modelirovaniya trekhmernikh dinamicheskikh zadach stroitelnoy aerogidrouprugosti, Dissertaciya na soiskanie uchenoy stepeni kandidata tekhnicheskikh nauk (2015).

5. Eurocode 1: Basis design and action on structures. Part 2-4: Wind action. ENV 19912-4, CEN (1994).

6. ANSYS 15.0 Help. 